\title{
Temporal Variations in Gas Temperature in an Atomization Stage of Cadmium and Tellurium Evaluated by Using the Two-line Method in Graphite Furnace Atomic Absorption Spectrometry
}

\author{
Haruki Shimabukuro, Tetsuya Ashino, and Kazuaki Wagatsuma ${ }^{\dagger}$ \\ Institute for Materials Research, Tohoku University, 2-1-1 Katahira, Sendai 980-8577, Japan
}

\begin{abstract}
In order to discuss the atomization process of an analyte element occurring in a graphite furnace for atomic absorption spectrometry, we measured variations in the characteristic temperature with the progress of an atomization stage, by using a two-line method under the assumption of a Boltzmann distribution. For this purpose, iron was chosen as the analyte element. Also, the atomic absorption of two iron atomic lines, Fe I $372.0 \mathrm{~nm}$ and Fe I $373.7 \mathrm{~nm}$, was simultaneously monitored as a probe for the temperature determination. This method enables variations in the gas temperature to be directly traced, yielding a temperature distribution closely related to the diffusion behavior of the probe element in the furnace. This temperature variation was very different from the furnace wall temperatures, which were monitored in conventional temperature control for atomic absorption spectrometry. Correlations between the gas temperature and the charring/atomizing temperatures in the heating program of the furnace were investigated. The atomization of cadmium and tellurium was also investigated by a comparison between the gas temperature with the wall temperature of the furnace. The atomic absorption of cadmium or tellurium appeared to be apart from the absorption of iron while the gas temperature was still low. Therefore, the analyte atoms could be atomized through direct contact with the wall of the graphite furnace, which has a much higher temperature compared to the gas atmosphere during atomization. Their atomization would be caused by conductive heating from the furnace wall rather than by radiant heating in the furnace.
\end{abstract}

(Received June 9, 2008; Accepted July 22, 2008; Published September 10, 2008)

\section{Introduction}

Graphite-furnace atomic absorption spectrometry (GF-AAS) ${ }^{1}$ is extensively employed for the determination of trace amounts of metallic elements in various application fields; for instance, industrial analysis, ${ }^{2,3}$ geological survey, ${ }^{4,5}$ and environmental assessment. $^{6-8}$ GF-AAS enables several elements, such as $\mathrm{Pb}$, $\mathrm{Cd}, \mathrm{Zn}$ and $\mathrm{Hg}$, to be determined at ultra-trace levels of $10^{-7 \%}$, with better detection sensitivity compared to the other analytical methods. ${ }^{1}$ In metallurgical processes, trace amounts of these elements, in some cases, exert a negative influence on the quality of manufactured materials; for example, their segregation at grain boundaries or their precipitation may cause cracking during hot rolling in steel materials. Furthermore, they have a harmful effect against the environment around human beings. GF-AAS can thus be an effective analytical method for industrial uses as well as environmental assessment. ${ }^{2-8}$

A graphite furnace for GF-AAS is electro-thermally heated to generate atomic vapor for the absorption measurement, along with a suitable temperature program for analyte elements to be pre-determined. Therefore, a temperature program, which usually comprises drying, charring, atomizing and cleaning stages, plays an important role for obtaining analytical values with higher sensitivity as well as better precision. ${ }^{1}$ In commercial GF-AAS, a radiation thermometer or a thermocouple device is used to control the heating of the furnace

$\dagger$ To whom correspondence should be addressed.

E-mail: wagatuma@imr.tohoku.ac.jp with the temperature program. However, the temperature estimated with these measuring devices corresponds to that of the wall of the graphite furnace; it is not the gas temperature in the furnace. The gas temperature can be considered to be of prime importance in controlling the atomizing process occurring in the furnace.

A direct measurement of the gas temperature in GF-AAS cannot be obtained with a radiation thermometer, principally due to difficulty in measuring transient variations in the atmosphere temperature inside the furnace. Only spectroscopic methods can be applied to obtain such information during heating of the furnace, due to their ability of remote sensing as well as rapid response. A two-line method, where a characteristic temperature is estimated from atomic absorption for two spectral lines having different lower energy levels, can provide a gas temperature under the thermodynamic equilibrium condition. ${ }^{9}$ Several papers have been published on the gas temperature estimated by atomic absorption with the two-line method. ${ }^{10-17}$ Terui et al. reported on the effectiveness of the twoline method for atomic absorption spectrometry, ${ }^{15}$ and also investigated the role of a matrix modifier related to temperature changes in a graphite furnace. ${ }^{16}$ Hirano et al. studied the gas temperature of arsenic in a graphite furnace and the variation when several modifier elements were added. ${ }^{17}$

We are interested in temporal variations in the gas temperature during the atomizing process occurring in a graphite furnace under the actual operating procedure, which can be in-situ monitored by using the two-line method, because they can yield knowledge on the diffusion of analyte elements occurring in the atomization process, as well as the temperature distribution in 
the furnace in the analytical applications. In this paper, the gas temperature was observed by using two iron atomic lines assigned to the $3 d^{6} 4 s-3 d^{5} 4 s 4 p$ transition when iron was employed as a probe element. Each absorption was monitored and then converted into the characteristic temperature under the assumption of a Boltzmann distribution. Atmosphere temperatures inside the furnace were investigated when the iron concentration as well as the charring and atomizing temperatures were varied as experimental parameters in heating the furnace. Furthermore, atomization stages of cadmium and tellurium were investigated in correlation with the gas temperature, by monitoring the iron lines and a cadmium or a tellurium atomic line simultaneously, so that the atomization process of cadmium or tellurium can be discussed.

\section{Experimental}

\section{Apparatus}

A simultaneous multi-element atomic absorption spectrometer (Z-9000, Hitachi Corp., Japan) with an auto-sampler system was employed, which can be equipped with four individual hollow cathode lamps as the light source. In this study, two iron hollow cathode lamps (Hamamatsu Photonics Corp., Japan) were installed to monitor two different wavelengths of the iron atomic line. The discharge current was set to be $5.0 \mathrm{~mA}$. A background correction was conducted by a dc-polarized Zeeman effect. The graphite furnace employed was a non-pyrocoating tube without a platform (Part No. 180-7400, Hitachi Corp., Japan).

\section{Reagents and procedure}

An iron stock solution of $2.0 \mathrm{~g} / \mathrm{L}$ was prepared by dissolving pure metallic iron (99.9\%) in solutions containing $7 \mathrm{M}$ nitric acid. It was further diluted with deionized water for working samples. The temperature program during heating the furnace was optimized using a pure iron solution of $3 \times 10^{-4}$-g/L concentration. The drying stage was carried out at $353-423 \mathrm{~K}$ for $30 \mathrm{~s}$ and the cleaning stage at $3273 \mathrm{~K}$ for $10 \mathrm{~s}$, and the charring and the atomizing stages were conducted at various temperatures to investigate their correlations with the gas atmosphere temperature. In the drying, charring, and cleaning stages, argon gas was introduced into the furnace at a flow rate of about $200 \mathrm{~mL} / \mathrm{min}$; however, no gas flow was required only in the atomization stage to keep the analyte species and thus to obtain a better sensitivity.

Cadmium and tellurium stock solutions of $1.0 \mathrm{~g} / \mathrm{L}$ were prepared by dissolving pure metals (99.9\%) with 7 M-nitric acid. They were further diluted with deionized water for working samples containing iron of $1.2 \times 10^{-3} \mathrm{~g} / \mathrm{L}$ and cadmium of $5 \times 10^{-5}-1 \times 10^{-6} \mathrm{~g} / \mathrm{L}$ (or tellurium of $1 \times 10^{-3}-6 \times 10^{-5} \mathrm{~g} / \mathrm{L}$ ). The temperature programs during heating the furnace were optimized for pure cadmium or tellurium when a cadmium solution $3 \times 10^{-6} \mathrm{~g} / \mathrm{L}$ or a tellurium solution of $1.8 \times 10^{-4} \mathrm{~g} / \mathrm{L}$ was employed. The drying stage was carried out at $353-423 \mathrm{~K}$ for $30 \mathrm{~s}$ and the cleaning stage at $3273 \mathrm{~K}$ for $10 \mathrm{~s}$. The charring temperature was determined at $923 \mathrm{~K}$ for cadmium and at $973 \mathrm{~K}$ for tellurium, because they began to be vaporized in the charring stage at higher temperatures. Each charring was conducted by heating the furnace up to the corresponding charring temperature required for $30 \mathrm{~s}$ followed by holding the temperature for $30 \mathrm{~s}$. An atomizing temperature of $3073 \mathrm{~K}$ was employed so that iron added as the temperature probe could be sufficiently atomized.

The absorption values for each spectral line were recorded on a personal computer through an analogous-to-digital converter at an interval of $0.02 \mathrm{~s}$, and the temporal variations in the gas temperature could be estimated directly using Eq. (4).

\section{Two-line method}

This method is based on the difference in the number density between two energy levels, which can be determined by a characteristic temperature under thermodynamic equilibrium. A graphite furnace is considered to be in local thermodynamic equilibrium (LTE), because the kinetic energy of particles in the furnace atmosphere can be averaged through their collisions occurring in the ambient pressure condition actively.

The following is a general formula for atomic absorption: ${ }^{18}$

$$
I_{\mathrm{abs}}=I_{0} N B h v l,
$$

where $I_{\mathrm{abs}}$ and $I_{0}$ mean the intensity of the light source after and before an absorption that occurs in a spectral line having a wavenumber of $v, N$ is the number density of an energy level related to the absorption, $l$ is the path length of the absorption layer, $B$ means an Einstein coefficient for induced absorption, and $h$ is the Planck constant. The ratio in the number density between two energy levels with the number of 1 or 2 is expressed using the following Boltzmann equation:

$$
N_{1} / N_{2}=g_{1} / g_{2} \exp \left[\left(E_{2}-E_{1}\right) / k T\right],
$$

where $k$ is the Boltzmann constant, and $N, g$, and $E$ mean the number density, the statical weight, and the excitation energy of each energy level, respectively. $T$ is an equilibrium temperature which should coincide with the gas temperature in the graphite furnace under LTE condition.

The intensity ratio of the incident and absorbed radiation, $R=$ $I_{\text {abs }} / I_{0}$, is measured for a spectral line related to each energy level. The coefficient $B$ can be converted to an Einstein coefficient for spontaneous emission in a straight way, as follows: ${ }^{18}$

$$
A=\left(8 \pi h v^{3} / c^{3}\right) B,
$$

where $c$ is the velocity of light. A term expressed as $F=\lambda^{2} g A$, where $\lambda$ is the wavelength and $A$ is the Einstein coefficient for the spontaneous emission of a spectral line, is calculated using literature data for each analytical line. Therefore, using Eqs. (1) - (3), and the terms $R$ and $F$, a general formula yielding the temperature is obtained;

$$
\ln \left(R_{1} / R_{2}\right)+\ln \left(F_{2} / F_{1}\right)=\left(E_{2}-E_{1}\right) / k T,
$$

where $k$ is the Boltzmann constant. The second term of Eq. (4) becomes constant if the two analytical lines are determined.

In this study, a pair of iron atomic lines, Fe I $372.0 \mathrm{~nm}$ and Fe I $373.7 \mathrm{~nm}$, was employed as probe lines for the temperature measurement. The former line is assigned to the transition from the $3 d^{6} 4 s^{2}{ }^{5} D_{4}(0.0000 \mathrm{eV})$ to $3 d^{5} 4 \mathrm{~s} 4{ }^{5} F_{5}(3.3319 \mathrm{eV})$ levels having a $\mathrm{gA}$ value of $1.467 \times 10^{8} \mathrm{~s}^{-1}$, and the latter line to the transition from the $3 \mathrm{~d}^{6} 4 \mathrm{~s}^{2}{ }^{5} \mathrm{D}_{3}(0.0516 \mathrm{eV})$ to $3 \mathrm{~d}^{5} 4 \mathrm{~s} 4 \mathrm{p}{ }^{5} \mathrm{~F}_{4}$ $(3.3682 \mathrm{eV})$ levels having a $\mathrm{gA}$ value of $0.994 \times 10^{8} \mathrm{~s}^{-1}{ }^{19}$ The energy difference between the $3 d^{6} 4 s^{2}{ }^{5} D_{4}$ and the $3 d^{6} 4 s^{2}{ }^{5} D_{3}$ levels is the most important factor in determining the temperature. A temperature estimation based on the atomic absorption is effective on the assumption that the spectral width of the emission line from the hollow cathode lamp is much narrower than the absorption line width. In our apparatus, the Zeeman splitting might cause any mismatch between these wavelengths; however, in the case of the iron quintet levels, the absorption profiles are expected to become broad because they 


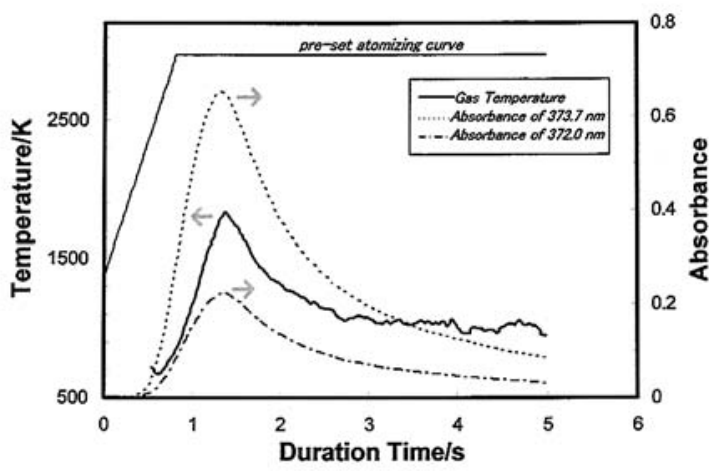

Fig. 1 Typical response of the gas temperature in an atomizing stage when the charring temperature is set at $1373 \mathrm{~K}$, together with variations in the absorbance of Fe I $372.0 \mathrm{~nm}$ and Fe I 373.7nm.

comprise lots of line components resulting from a complicated abnormal Zeeman effect. It should be noted that their Zeeman splittings as well as the uncertainty of the gA values determine the accuracy of the estimated temperatures.

\section{Results and Discussion}

Temporal changes in the gas temperature through the two-line method

Figure 1 shows a temporal change of the gas temperature in an atomization stage, which is estimated from the absorption of the iron spectral lines, Fe I $372.0 \mathrm{~nm}$ and Fe I $373.7 \mathrm{~nm}$, after the charring temperature of $1373 \mathrm{~K}$ is kept for $30 \mathrm{~s}$. In the sampling procedure, an iron solution of $1.2 \times 10^{-4} \mathrm{~g} / \mathrm{L}$ and an injection volume of $0.05 \mathrm{~mL}$ were employed. For a comparison, the programmed variation in the furnace temperature is also indicated using a straight line; further, changes in the absorbance value of these iron lines are added to the graph. In this atomization stage, the wall temperature was elevated from 1373 $\mathrm{K}$ (charring temperature) to $2973 \mathrm{~K}$ (atomizing temperature) for $1 \mathrm{~s}$, and was then held at $2973 \mathrm{~K}$. The absorption for these iron lines began to be observed from a duration time of $c a .0 .6 \mathrm{~s}$ and was then greatly reduced towards the end of the atomization stage; therefore, the gas temperature could be obtained only for a period of $0.6-4.2 \mathrm{~s}$.

The gas temperature is elevated with the progress of the atomization, and reaches a maximum $(c a .1800 \mathrm{~K})$ after a duration time of $1.4 \mathrm{~s}$. The gas temperature seems not to be elevated at the initial stage of the atomization, although the furnace temperature is held at $1373 \mathrm{~K}$ during the charring stage. This effect is because argon gas flowing in the furnace prevents the gas temperature from being elevated. It is interesting to note that the maximum of the gas temperature is much lower than the wall temperature, and that it becomes reduced down to 1100 $1200 \mathrm{~K}$ at the latter stage of the atomization, whereas the furnace wall is maintained at $2973 \mathrm{~K}$. This phenomenon can be understood by considering a time lag between the wall and the atmosphere temperatures, the temperature distribution in the furnace, and the spatial distribution of iron atoms varied with the development of atomization. Figure 2 illustrates a model expressing the production and subsequent diffusion of iron atoms in a graphite furnace. Radiant heating of the furnace gas would not follow a rapid change in the wall temperature occurring for a short ramp time, which generally explains the disagreement between the wall and the gas temperatures. The

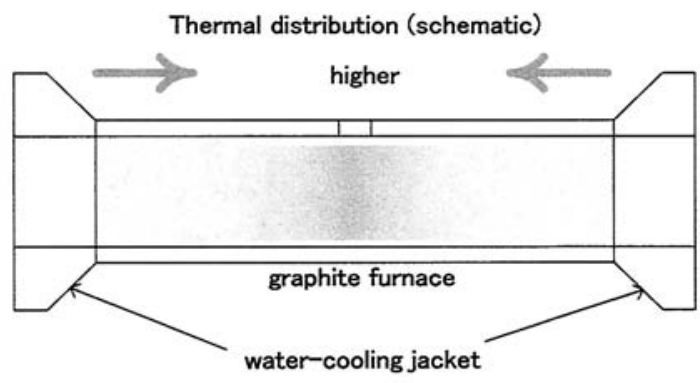

(a)

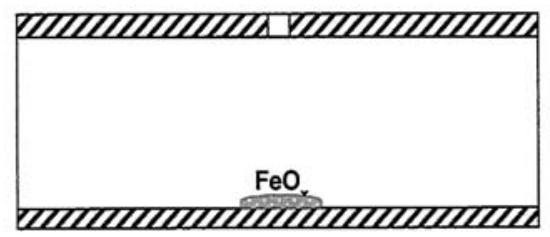

(b)

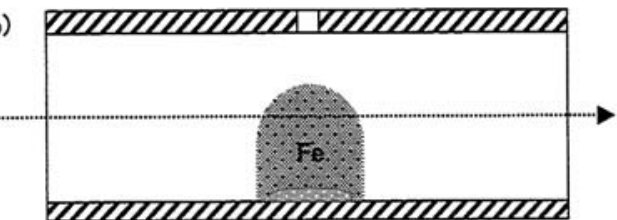

(c)
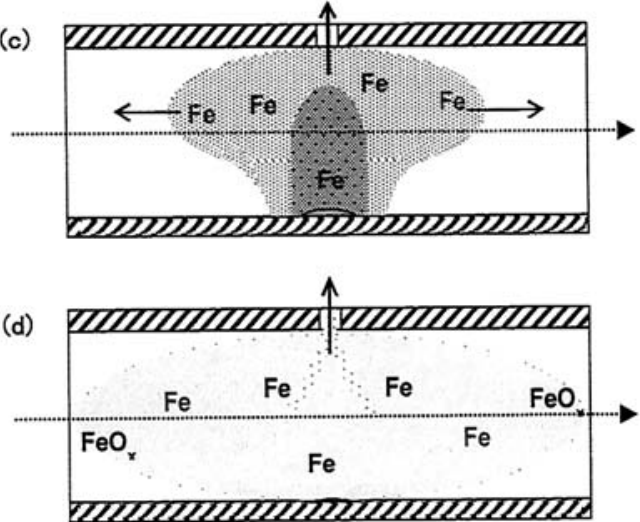

Fig. 2 Schematic drawing of the thermal distribution of a graphite furnace and the progress of the atomization of iron atoms from (a) to (d).

charred sample can be atomized directly by the wall temperature because it is contacted with the inner wall of the furnace, whereas the gas is heated indirectly. In addition, the wall should have a lower temperature at the edge of the furnace compared to the central portion because the graphite furnace is installed with a water-cooled jacket, thereby leading to a similar distribution in the atmosphere temperature; the gas has a lower temperature at the edge of the furnace. When the atomization stage lasts as shown in Fig. 2(d), atomized iron atoms may diffuse towards the edge portion so that some of these atoms absorb the radiation repeatedly, meaning that these iron atoms are excited at the lower temperature of the edge position. As a result, the gas temperature tends to decrease at the latter stage of atomization, as can be seen in Fig. 1.

\section{Effect of the iron concentration}

Figure 3 shows temporal changes of the gas temperature in the atomization stage when the iron concentration is varied from 0.3 $\times 10^{-3}$ to $1.2 \times 10^{-3} \mathrm{~g} / \mathrm{L}$. In this case, the atomizing temperature was fixed at $2973 \mathrm{~K}$, after the charring stage had been conducted 


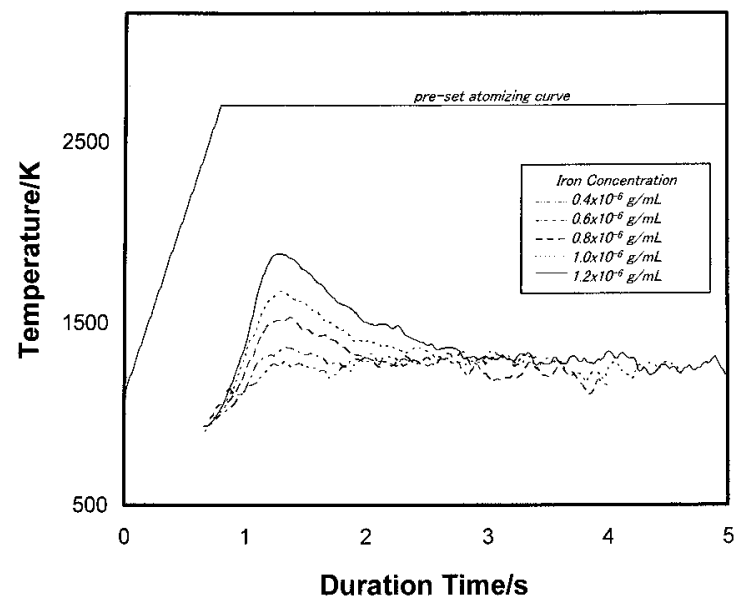

Fig. 3 Variation of the gas temperature in the atomization stage when samples having different iron concentration are introduced.

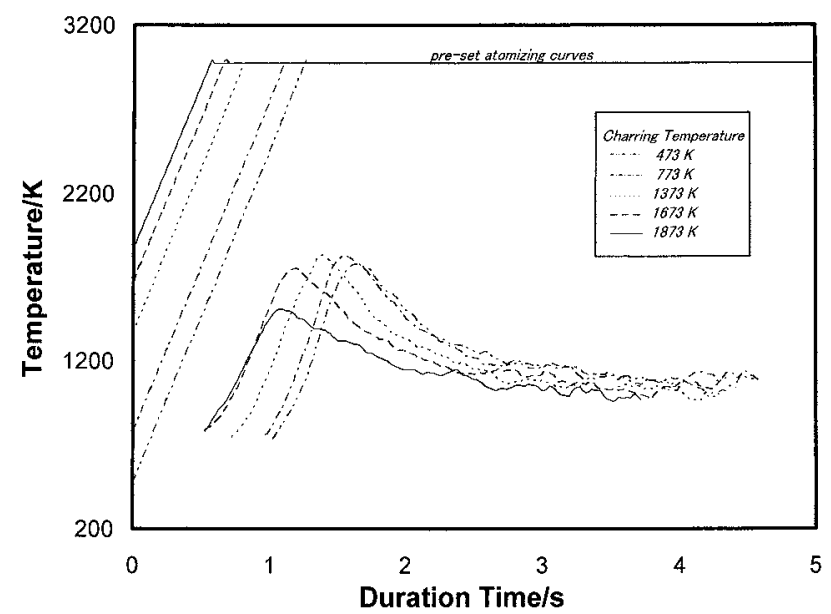

Fig. 4 Variation of the gas temperature in the atomization stage when the furnace is preheated at different charring temperatures.

at a temperature of $1373 \mathrm{~K}$ for $30 \mathrm{~s}$. Independent of the iron concentration, the gas temperatures are almost the same at the initial stage of atomization, which appears to be $c a .970 \mathrm{~K}$ at a duration time of $0.8 \mathrm{~s}$. The maximum peak of the gas temperature appears at a duration time of $1.4 \mathrm{~s}$ when the iron concentration is more than $0.8 \times 10^{-3} \mathrm{~g} / \mathrm{L}$, although the maximum peak disappears at lower iron concentrations, which probably results from an increase in the maximum temperature with increasing iron concentration in the furnace. It is considered that the temperature distribution of the furnace wall is not altered by the analyte concentration since the temperature program is exactly the same. Therefore, this phenomenon would relate not to changes in the wall temperature but to a diffusion of the iron atoms in the atomization process. Smaller amounts of iron species can be atomized and then move towards the edge portion of the furnace at a shorter duration time, while the atmosphere is not heated sufficiently. A longer duration time would be required to atomize larger amounts of iron species, leading to stay of some iron atoms in the central portion of the furnace having higher temperatures.

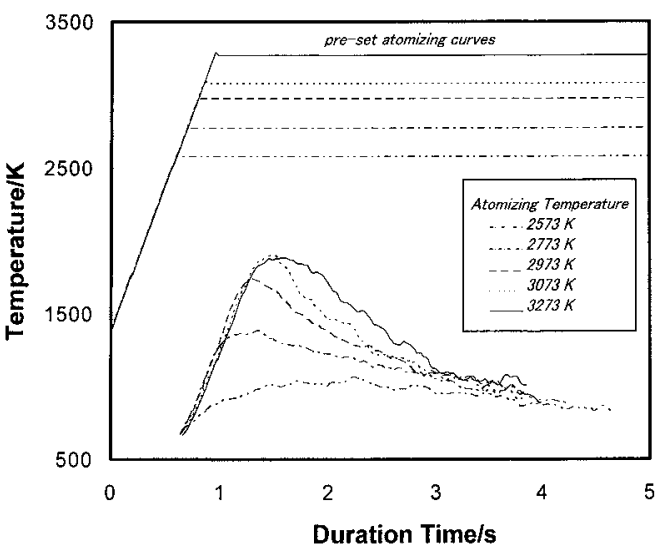

Fig. 5 Variation of the gas temperature in the atomization stage when different atomizing temperatures are selected.

\section{Effect of charring temperature}

Figure 4 shows temporal changes of the gas temperature in the atomization stage when the charring temperature is varied from 473 to $1873 \mathrm{~K}$. In this case, the atomizing temperature was fixed at $2973 \mathrm{~K}$ and $0.05 \mathrm{~mL}$ of the $1.2 \times 10^{-3} \mathrm{-g} / \mathrm{L}$ iron solution was injected as the sample. The maximum peak of the gas temperature appears at a longer duration time along with decreasing charring temperature, and the maximum temperature decreases in the range of the charring temperature from 1373 to $1873 \mathrm{~K}$. The shift in the maximum peak can be explained from a difference in the atomization step; as indicated as straight lines in Fig. 4, the time required for the steady atomization stage $(2973 \mathrm{~K})$ is only $0.6 \mathrm{~s}$ at a charring temperature of $1873 \mathrm{~K}$ whereas the corresponding time becomes $1.3 \mathrm{~s}$ at $473 \mathrm{~K}$, leading to a delay in atomization of the iron sample. The rapid atomization, which is expected to occur at higher charring temperatures, could result in a rapid diffusion of the iron species, while the gas atmosphere in the furnace is not heated sufficiently. This effect leads to a decrease in the gas temperature at higher charring temperatures.

\section{Effect of atomizing temperature}

Figure 5 shows temporal changes of the gas temperature in the atomization stage when the atomizing temperature is varied from 2573 to $3273 \mathrm{~K}$. In this case, the charring condition was conducted at $1373 \mathrm{~K}$ for $30 \mathrm{~s}$, and $0.05 \mathrm{~mL}$ of the $1.2 \times 10^{-3}-\mathrm{g} / \mathrm{L}$ iron solution was introduced. Larger maximum peaks of the gas temperature move towards a longer duration time along with increasing atomizing temperature; for example, the maximum temperature of $1870 \mathrm{~K}$ appears at a duration time of $1.6 \mathrm{~s}$ when the atomizing temperature is $3273 \mathrm{~K}$, whereas the corresponding temperature is $1370 \mathrm{~K}$ at a duration time of $1.2 \mathrm{~s}$ when the atomizing temperature is $2773 \mathrm{~K}$. In this case, it is expected that the furnace atmosphere is more heated at higher atomizing temperatures; in fact, the gas temperature was elevated as shown in Fig. 5. Also, the appearance of the maximum peak would be determined by an expansion of the area having each steady gas temperature when the atomizing temperature is elevated. No distinct peak of the gas temperature was observed at an atomizing temperature of $2573 \mathrm{~K}$, probably due to a delay of the atomization because it is not caused sufficiently at this low atomizing temperature.

\section{Absorption of cadmium}

Figure 6 shows temporal changes in the absorbance for the 


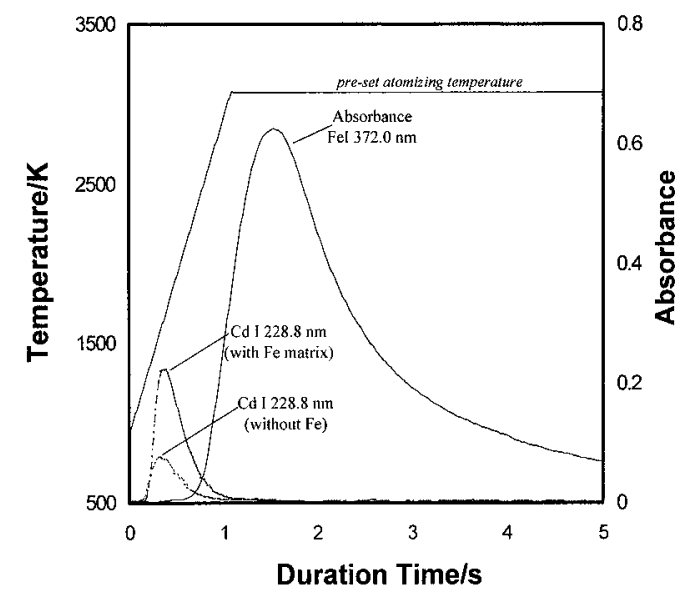

Fig. 6 Transient signals of the absorbances of Fe I $372.0 \mathrm{~nm}$ and $\mathrm{Cd}$ I $228.8 \mathrm{~nm}$ in an atomization stage after the charring at $1073 \mathrm{~K}$ for $30 \mathrm{~s}$. The absorbance of the Cd I line is monitored with/without an iron matrix.

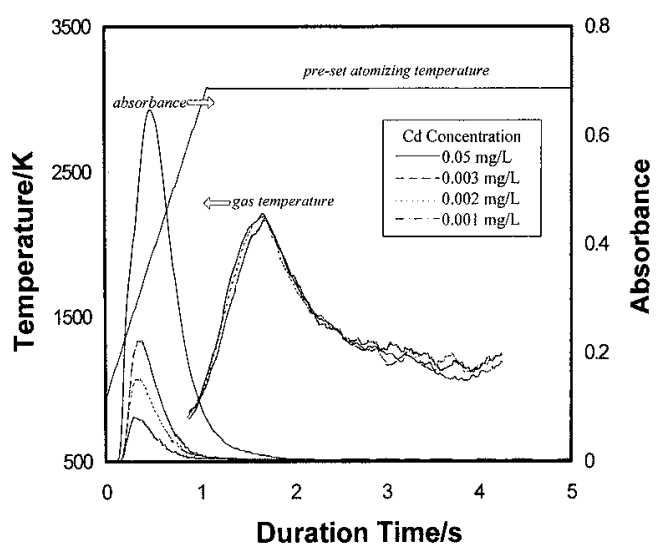

Fig. 7 Variation of the gas temperature in the atomization stage, together with the absorbance of $\mathrm{Cd} \mathrm{I} 228.8 \mathrm{~nm}$, when the concentration of cadmium is varied.

iron and cadmium lines in an atomization stage. In this case, the atomizing temperature was fixed at $3073 \mathrm{~K}$, after the charring temperature of $1073 \mathrm{~K}$ was kept for $30 \mathrm{~s}$. The absorbances of cadmium were measured when the iron element was in the sample solution or absent. It is found in Fig. 6 that the cadmium signal appears much earlier than the iron signal; the cadmium absorbance has a maximum at a duration time of $0.4 \mathrm{~s}$, which is on the way of the heating to the atomization temperature (3073 $\mathrm{K})$, whereas the iron peak appears after the atomization reaches the steady stage at a duration time of $1.7 \mathrm{~s}$. This effect can be understood by the difference in the thermal properties between cadmium and iron; cadmium can be atomized at a lower wall temperature because cadmium compounds are easily decomposed as well as evaporated due to the low boiling temperature. It has been reported that the iron element acts as a modifier in the cadmium determination, ${ }^{11}$ meaning that the detection sensitivity of cadmium can be improved in the coexistence of iron. Also, in our experiment the absorbance of the cadmium line became elevated compared to that of the ironfree sample, as shown in Fig. 6. However, peaks of the cadmium absorbance appear at almost the same duration time,

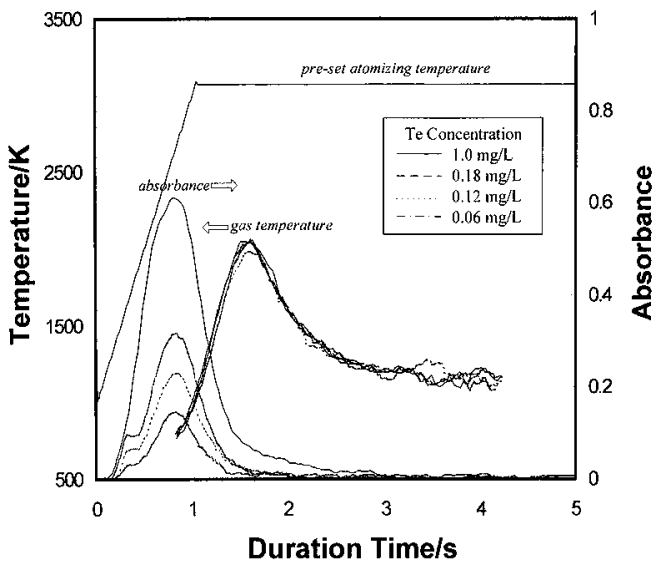

Fig. 8 Variation of the gas temperature in the atomization stage, together with the absorbance of Te I $214.3 \mathrm{~nm}$, when the concentration of tellurium is varied.

regardless of the iron matrix. This observation implies that iron and cadmium atoms are atomized individually according to each thermal property; thus, in the atomization stage, the iron matrix does not keep the cadmium species at the higher temperatures.

Figure 7 shows variations in the gas temperature, which is estimated from the absorption of the iron spectral lines, and changes in the cadmium absorbance as a function of the duration time in an atomization stage, when the cadmium concentration is varied from $1 \times 10^{-6}$ to $5 \times 10^{-5} \mathrm{~g} / \mathrm{L}$ in a coexistence of $1.2 \times$ $10^{-4}-\mathrm{g} / \mathrm{L}$ iron. An injection volume of $0.05 \mathrm{~mL}$ was employed for each measurement. Temporal changes in the gas temperature were almost the same in the atomization stage, regardless of the cadmium concentration in the sample; a maximum of the gas temperature appeared at a duration time of $1.7 \mathrm{~s}$ and the temperature was $c a .2200 \mathrm{~K}$, indicating that the atomization conditions became unchanged. It is further found in Fig. 7 that an absorbance peak of cadmium is little moved at a duration time of $0.4 \mathrm{~s}$ when the cadmium concentration is changed, although the absorbance increases along with the cadmium concentration. The gas temperature is expected to be fairly low (less than $700 \mathrm{~K}$ ) at the duration time when the cadmium peaks appear, where an accurate value cannot be estimated from this two-line method because the absorption of the iron lines is very weak at the initial stage of atomization. It is thus considered that the vaporization of cadmium is determined by the wall temperature rather than the gas temperature, and therefore collisional processes occurring in the gas atmosphere would contribute less to atomization.

\section{Absorption of tellurium}

Figure 8 shows temporal changes in the gas temperature as well as the absorbance of the tellurium line, when the tellurium concentration is varied from $1 \times 10^{-6}$ to $5 \times 10^{-5} \mathrm{~g} / \mathrm{L}$ in a coexistence of $1.2 \times 10^{-4} \mathrm{~g} / \mathrm{L}$ iron. This graph is similar to the case of cadmium, as indicated in Fig. 6, except that the absorbance maximum is slightly shifted towards a longer duration time (ca. $0.8 \mathrm{~s})$. As a result, we could estimate that the gas temperature when the maximum peaks appear was $c a$. $800 \mathrm{~K}$. This difference in the absorbance maximum between cadmium and tellurium would be because the boiling point of tellurium is slightly higher compared to cadmium: $1038 \mathrm{~K}$ for cadmium and $1263 \mathrm{~K}$ for tellurium. Also, in the case of tellurium, temporal variations of the gas temperature are less 
effected by the analyte concentration, and they are almost the same as the variations for cadmium having a maximum peak at a duration time of $1.7 \mathrm{~s}$. Accordingly, similar to the discussion for cadmium, the vaporization of tellurium is determined by the wall temperature rather than the gas temperature.

\section{Conclusions}

The gas temperature is estimated with the two-line method using iron atomic lines when the sample concentration, the charring temperature, and the atomizing temperature are varied as experimental parameters. The gas atmosphere temperature at the atomization stage is much lower than the atomizing temperature in the temperature program. This indicates a distinct difference between the wall temperature of the furnace, which is monitored for control in the temperature program of GF-AAS, and the atmosphere temperature. Also, changes in the gas temperature is determined not only by the temperature distribution, but by the spatial distribution of iron atoms through their diffusion in the atomization stage.

The maxima of the cadmium and tellurium absorbances appear in the initial stage of atomization, where the gas atmosphere is not heated sufficiently, and thus has a lower temperature. This phenomenon implies that the analyte atoms are atomized through direct contact with the wall of the graphite furnace, which has a much higher temperature compared to the gas atmosphere during atomization. Therefore, the atomization of cadmium and tellurium can be understood based on changes in the wall temperature, which is monitored for control in the temperature program of GF-AAS, rather than changes of the gas temperature.

The two-line method is a remote-sensing technique; thus, it can provide useful information on various transient processes occurring in the graphite furnace in GF-AAS.

\section{Acknowledgements}

The authors gratefully acknowledge financial support by a grant from Steel Industry Foundation for the Advancement of Environment Protection Technology, Japan.

\section{References}

1. J. A. Broekaert, "Analytical Atomic Spectrometry with Flames and Plasmas", 2002, Wiley-VCH Verlag, Weinheim.

2. T. Ashino and K. Takada, J. Anal. At. Spectrom., 1966, 11, 577.

3. T. Ashino and K. Takada, Anal. Chim. Acta, 1995, 312, 157.

4. Y. Tamari, H. Tsuji, and Y. Kusaka, Bunseki Kagaku, 1984, 33, T95.

5. Z. Grobenski, D. Weber, B. Wetz, and J. Wolff, Analyst, 1983, 108, 925.

6. R. E. Sturgeon, Spectrochim. Acta, Part B, 1997, 52B, 1451.

7. J. Ohyama, F. Maruyama, and Y. Dokiya, Anal. Sci., 1987, $3,413$.

8. K. Itoh, K. Akatsuka, and I. Atsuya, Bunseki Kagaku, 1984, 33,301

9. P. W. J. M. Boumans, "Theory of Spectrochemical Excitation", 1966, Prenum Press, New York.

10. R. F. Browner and J. D. Winefordner, Anal. Chem., 1972, 44, 247

11. W. M. G. T. van den Broek, L. de Galan, J. P. Matousek, and E. J. Czobik, Anal. Chim. Acta, 1978, 100, 121.

12. K. Kitagawa, Y. Ide, and T. Takeuchi, Anal. Chim. Acta, 1980, 113, 21.

13. C. L. Chakrabarti, S. Wu, R. Karwowska, J. T. Rogers, L. Haley, P. C. Bertels, and R. Dick, Spectrochim. Acta, Part $B, \mathbf{1 9 8 4}, 39 B, 415$.

14. D. D. Siemer and W. Frech, Spectrochim. Acta, Part B, 1984, 39B, 261.

15. Y. Terui, K. Yasuda, and K. Hirokawa, Anal. Sci., 1991, 7, 599.

16. Y. Terui, K. Yasuda, and K. Hirokawa, Anal. Sci., 1991, 7, 593.

17. Y. Hirano, K. Yasuda, and K. Hirokawa, Anal. Sci., 1994, 10,481 .

18. S. Minami and S. Musha (ed.), "Atomic Spectrometric Analysis", Part 1, 1979, Maruzen, Tokyo.

19. J. R. Fuhr, G. A. Martin, and W. L. Wiese, J. Phys. Chem. Ref. Data, 1998, 17, Suppl. 4. 